\title{
National standards of length for high-capacity optical fiber communication systems
}

\author{
— Development of fiber-based optical frequency combs - \\ Hajime INABA* , Atsushi OnAE and Feng-Lei Hong \\ [Translation from Synthesiology, Vol.7, No.2, p.68-80 (2014)]
}

\begin{abstract}
An optical frequency comb is a collection of laser modes with identical frequency intervals in the visible to near-infrared regions that enables direct comparison of optical frequencies with microwave atomic frequency standards. Traditional solid-state laser-based frequency comb systems are large, expensive, and very difficult to operate for long periods of time during experiments. From an early stage of development, we proposed fiber lasers as a feasible means of achieving a reliable frequency comb. After we succeeded in developing an in-house fiber-based frequency comb at the National Institute of Advanced Industrial Science and Technology (AIST), we made further advances, including calibration of the optical telecommunication band, establishment of national standards of length, and development of a narrow-linewidth comb for optical lattice clocks.
\end{abstract}

Keywords : Optical frequency comb, fiber-based frequency comb, optical frequency measurement, length standard, optical telecommunication band, wavelength-stabilized lasers

\section{Introduction}

Before the invention of the optical frequency comb, frequency measurement in the optical domain was extremely difficult. Requiring a number of microwave oscillators, special frequency multipliers/mixers, and wavelength or frequencystabilized lasers (hereinafter referred to as "stabilized lasers") as measuring devices, the "frequency chain," ${ }^{[1][2]}$ which linked with frequency in the optical domain after repeatedly multiplying and mixing in sequence, was based on 9192 $631770 \mathrm{~Hz}$, the microwave frequencies generated from the cesium atomic frequency standard. The extremely extensive measuring equipment required not only development but also enormous costs and human resources. In addition, this equipment could only measure the frequency of a single laser, which meant that lasers with different wavelengths required different frequency chains to be constructed.

Under these circumstances, the International Committee for Weights and Measures (CIPM) prepared a list of frequencystabilized lasers based on the laser frequencies measured with frequency chains and recommended that they be used as wavelength standards (length standards) for the purpose of a practical metric realization. ${ }^{[3]}$ Frequency-stabilized lasers developed in national metrology institutes of different nations are gathered to validate their equivalence for the international comparison of frequencies (hereafter referred to as "international comparison"). The lasers compared internationally serve as the basis for the reference laser for length measurement in a nation. The most internationally compared laser is the $633 \mathrm{~nm}$ iodine-stabilized He-Ne laser.
In Japan, the national standard of length had been the iodinestabilized He-Ne laser kept by the National Institute of Advanced Industrial Science and Technology (AIST) until 2009. ${ }^{[4]}$

Frequency measurement in the optical domain has a number of applications other than the length standard, among which the most important field in a social context is optical fiber communications. In the 1990s, wavelength-division multiplexing began to increase communication capacity, and many specialists anticipate that precise optical frequency management is required in the near future. It was therefore necessary to add a stabilized laser in the $1.5-\mu \mathrm{m}$ optical communication band as a frequency standard to the CIPM's recommendation, and to develop the technology to measure laser frequency in the 1.5- $\mathrm{m}$ band. In Japan, where the 633$\mathrm{nm}$ iodine-stabilized He-Ne laser served as the national measurement standard, the $1.5-\mu \mathrm{m}$ stabilized lasers needed to be traceable to the $633-\mathrm{nm}$ national measurement standard.

It was in 1999 that the first breakthrough came in overcoming the difficulties in conventional optical frequency measurement using the frequency chain. German and American groups achieved absolute measurement of laser frequency using the "optical frequency comb" with a mode-locked laser, ${ }^{[5][6]}$ which led to enormous technical innovations in this field. The optical frequency comb was a great success and enabled laser frequency to be measured to the accuracy of the cesium atomic frequency standard (11 to 16 digits depending on the mean time or oscillator types). The linkage of optical frequency with microwave frequency by means of the optical frequency comb

National Metrology Institute of Japan, AIST Tsukuba Central 3, 1-1-1 Umezono, Tsukuba 305-8563, Japan

* E-mail: h.inaba@aist.go.jp

Original manuscript received May 30, 2013, Revisions received October 4, 2013, Accepted October 24, 2013 
(in a comparatively easy manner) gave great momentum to the study of the "optical clock" as a next-generation frequency standard to replace the cesium atomic clock. J. L. Hall and T. W Hänsch, who had been engaged in the invention of the optical frequency comb, were awarded the Nobel Prize for Physics in 2005 in recognition of their "contributions to the development of laser-based precision spectroscopy, including the optical frequency comb technique".

However, this does not mean that their invention solved all problems. To use the optical frequency comb as a practical tool in various applications, a second breakthrough is required to overcome problems in the photonic crystal fiber or the titanium-sapphire (Ti:S) laser, which was the mode-locked laser used for the optical frequency comb at that time. Large in size, the Ti:S laser requires an expensive and power-consuming pump laser. In addition, the complicated equipment needs an operator with technical knowledge for operation of the optical frequency comb. With difficulties in continuous operation over a long period of time, even in a laboratory environment, it still has a long way to go before it can be commercialized.

This study is related to the second breakthrough noted above and focuses on the development of a reliable optical-fiber frequency comb using an erbium-doped fiber-based modelocked laser (hereinafter referred to as a "fiber comb") as an alternative to the conventional optical frequency comb using a mode-locked Ti:sapphire laser-based frequency comb (hereinafter referred to as a "Ti:S comb"). This paper begins with a brief explanation of the principles of the optical frequency comb and the problems of the Ti:S comb. It then provides an overview of using the fiber comb to solve these problems, as well as giving background of R\&D including the fabrication of the comb. Furthermore, this paper describes frequency measurement of the optical communication band laser, national measurement standards for length including international activities, and a high-speed controllable frequency comb for next-generation optical frequency standards as achievements of this development.

\section{Optical frequency comb}

To understand the optical frequency comb, it is necessary to consider both waveforms on the time domain and spectra on the frequency domain. As shown in Fig. 1, the optical frequency comb observed in the time domain is the ultrashort optical pulse train, which consists of optical pulses each with durations between several and several hundreds of femtoseconds arranged at regular time intervals. In the frequency domain, in contrast, it is the Fourier transform of the optical frequency comb, and appears as an assembly of line spectra arranged at regular frequency intervals. The broadened spectra and mode spacings in the frequency domain represent the reciprocals of the optical pulse sharpness (duration) and interval time of the optical pulse train in the time domain, respectively. It is also possible to determine that each line spectrum on the frequency domain is continuous light and that their phases are synchronized to form optical pulses as a whole.

The most important characteristic of the optical frequency comb is that the frequency spacing of line spectra is consistent regardless of wavelength. For instance, the vertical modes of optical cavities represented by the Fabry-Perot cavity have spectra very similar to those of the optical frequency comb, although the free spectral range $c / 2 n L$ (where $c$ stands for light speed, $n$ for phase refractive index, and $L$ for cavity length) varies with atmosphere or cavity dispersion (i.e., $n$ varies by wavelength). In contrast, the mode spacing of the optical frequency comb is consistent regardless of wavelength, as it is produced at regular intervals as a result of mode synchronization. As shown in Fig. 1 , the $N$-th comb mode

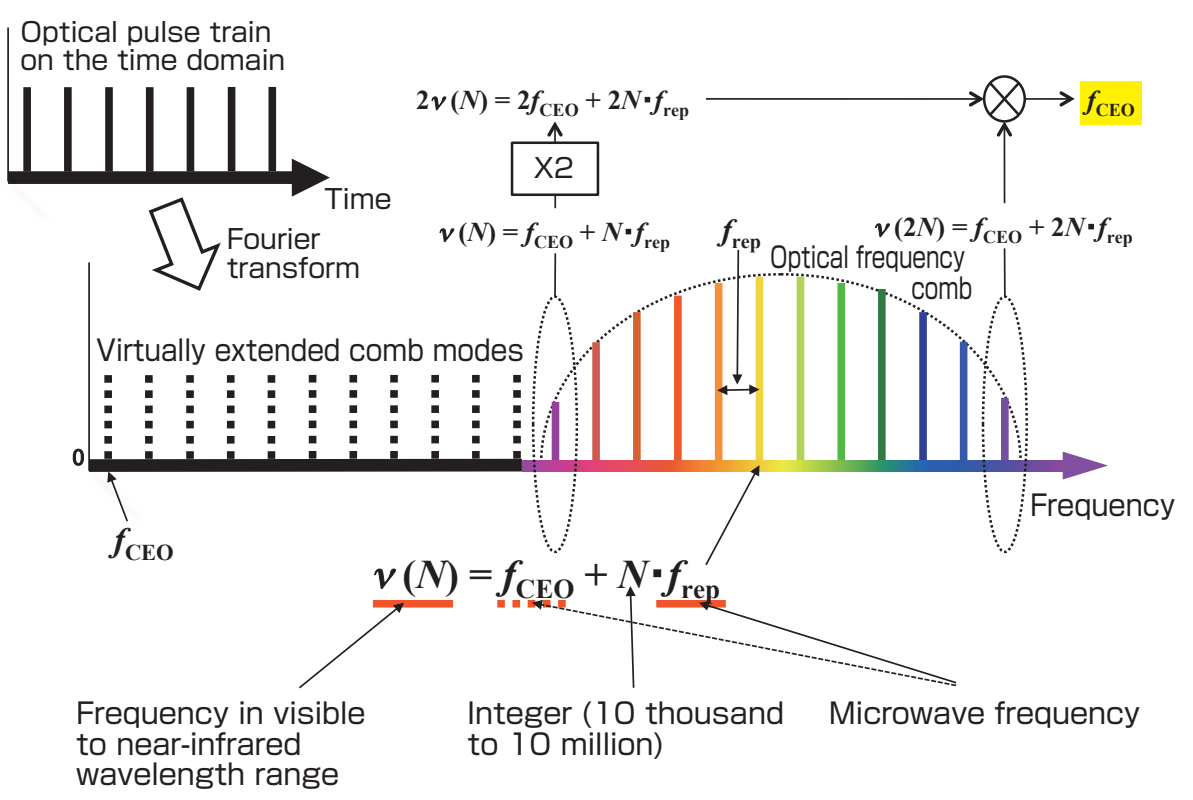

Fig. 1 Conceptual diagram of the optical frequency comb

The ultrashort optical pulse train observed in the time domain is Fourier-transformed and appears as the optical frequency comb in the frequency domain. With consistent mode spacing regardless of wavelength, it is appropriate to virtually extend the comb mode outside the actual comb mode. The radiofrequency $f_{\text {rep }}$ is uniquely linked to the optical frequency $v(N)$. Furthermore, broadening the frequency comb by more than an octave facilitates detection of the $f_{\text {CEO }}$ signal. 
frequency $v(N)$, regarding $f_{\text {CEO }}$ as the zeroth frequency, in the optical domain can thus be described as:

$v(N)=f_{\mathrm{CEO}}+N \cdot f_{\text {rep }}$

where $f_{\text {rep }}$ represents the frequency spacing between adjacent comb modes, equal to a repetition frequency of the ultrashort optical pulse train in the time domain; $N$ stands for an integer from several tens of thousands to several millions; and $f_{\text {CEO }}$ stands for a uniform offset frequency of the optical frequency comb from $N \cdot f_{\text {rep }}$ of each mode. This equation indicates that the unique $v(N)$ between 180 and $600 \mathrm{THz}$ (equivalent to the near-infrared to visible wavelength range) is determined by defining $f_{\text {CEO }}$ and $f_{\text {rep }}$ between several tens and several hundreds of $\mathrm{MHz}$ (hereinafter referred to as microwave frequency). In particular, it should be noted that the microwave frequency $f_{\text {rep }}$ is multiplied by an integer and reaches a frequency in the optical frequency range. The optical frequency comb could thus be regarded as a frequency multiplier (or divider) that links microwave frequency with optical frequency.

Values for $f_{\text {CEO }}$ are extremely small compared to an optical frequency of several hundreds of THz. However, $f_{\mathrm{CEO}}$ is an important parameter that links frequencies in the optical range to frequencies from atomic clocks in the microwave range. To detect $f_{\text {CEO }}$, the spectra of the optical frequency comb should be broadened by more than "an octave". Figure 1 shows how to observe $f_{\mathrm{CEO}}$. The "octave-spanning comb" means that the $N$-th and $2 N$-th modes simultaneously exist in the comb. The difference in frequency between the second harmonic of the $N$-th mode and the $2 N$-th mode is equal to $f_{\text {CEO }}$, which is how $f_{\text {CEO }}$ is observed in an experimental manner.
The output spectrum of the mode-locked laser is an optical frequency comb, but it is not generally broadened to an octave. In most cases, these optical spectra are broadened using the photonic crystal fiber or the highly nonlinear fiber. In doing so, the original comb mode is broadened by such nonlinear effects as self-phase modulation, four-wave mixing, and Raman amplification while maintaining frequency spacing. The "frequency ruler," with broadening to as much as an octave in the optical frequency range, is used in a number of applications including frequency metrology.

\section{Problems of the optical frequency comb using the Ti:S laser and solutions using the fiber comb}

The octave-spanning comb used a Ti:S laser as a modelocked laser, and a photonic crystal fiber for broadening the spectrum in the initial stage. Although the Ti:S laser realized the octave-spanning comb and was a major success, serious problems remained in commercializing them. This section describes some important problems of Ti:S combs that need to be solved and how fiber combs solve these issues.

\subsection{Large and expensive pump laser}

Figure 2 shows a schematic view of the Ti:S comb. A highpower solid-state laser is used to excite the Ti:S laser. The typical, commercially available laser head and controller are comparatively large in size, as shown in the photo. The main units of the pump laser and Ti:S laser require water coolers. In addition, the 5-10-W pump laser used for the Ti:S laser for the optical frequency comb is expensive, and the consumables that need to be replaced periodically are also

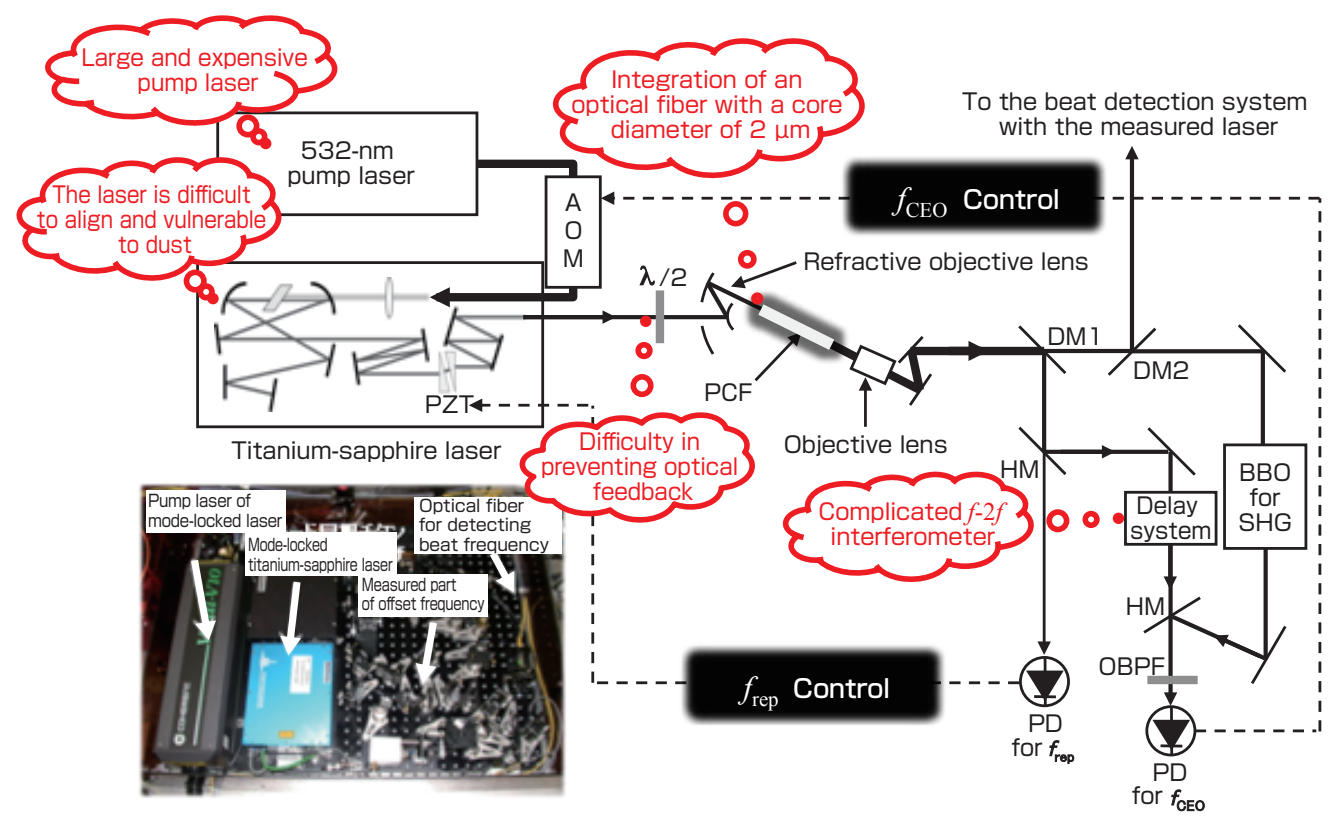

Fig. 2 Schematic view of the optical fiber comb system using the titanium-sapphire laser

The space optical system is hard to operate over a long period of time. The photo shows an example compact system. PZT: piezoelectric transducer; AOM: acousto-optic modulator; $\lambda / 2$ : 1/2-wavelength plate; PCF: photonic crystal fiber; DM: dichroic mirror; HM: half-transparent mirror; OBPF: optical bandpass filter; BBO: $\beta$ barium borate crystal; SHG: second-harmonic generation; PD: photo detector. 
expensive.

Figure 3 shows a schematic view of the fiber comb system, where the small semiconductor laser, which is incorporated into a butterfly package as shown in the bottom right photo, is used as a pump laser. The controller for the system is markedly smaller than that for the solid-state laser, which means that the entire system could be made much smaller than the Ti:S system. The price is less than a tenth of that for the solid-state laser used for exciting the Ti:S laser. The mode-locked fiber laser does not require water cooling.

\subsection{Difficulties in long-term continuous operation}

It is difficult to continuously operate the Ti:S comb over a long period of time. One of the reasons is instability of optical coupling to the photonic crystal fiber used for broadening the spectrum of the optical frequency comb. Since the core diameter of the photonic crystal fiber is as small as approximately $2 \mu \mathrm{m}$ (while the core diameter of the regular single-mode fiber is approximately $10 \mu \mathrm{m}$ ), the relative positions of laser light and the fiber core are likely to fluctuate as a result of temperature changes or other factors. In addition, the beam orientation of a large pump laser and a Ti:S laser tends to be unstable. As a result, the coupling efficiency of laser light to the fiber changes over time, and the $\mathrm{S} / \mathrm{N}$ ratios of the offset beat signal and beat signal with a continuous wave $(\mathrm{CW})$ laser are reduced, which lead to a loss of control and/or frequency counting. Hardly resistant to dust, the Ti:S laser, acting as a space laser, attracts a very small quantity of dust around the high-optical intensity crystal, resulting in unstable operation. Therefore, optical frequency measurement with the Ti:S comb is unlikely to be feasible over 24 consecutive hours.

In the fiber comb system, the mode-locked fiber laser, the optical amplifier, and the highly nonlinear fiber consist of optical fiber systems, enabling fusion splices between fibers. This eliminates the need for precise alignment that is essential for the space optical system and allows all connections with a single optical fiber, which provides an almost complete solution to the abovementioned drawbacks of the Ti:S comb.

\section{Fiber comb development}

As mentioned above, the developed fiber comb displayed obvious advantages. We began with the "initial assessment of the fiber combs" to ensure that it was feasible. We then formulated the idea that robust and low-noise fiber combs may be self-fabricated if such fundamental technological goals as "design and fabrication of the mode-locked fiber lasers," "design and fabrication of amplifiers," "assessment of the highly nonlinear fibers," and "development of the highspeed controllable frequency comb" are achieved. Successful self-fabrication can bring about an optical frequency comb that could achieve the aims of "calibration of laser frequency in the optical communication band," "development of national measurement standards," "applications to the optical

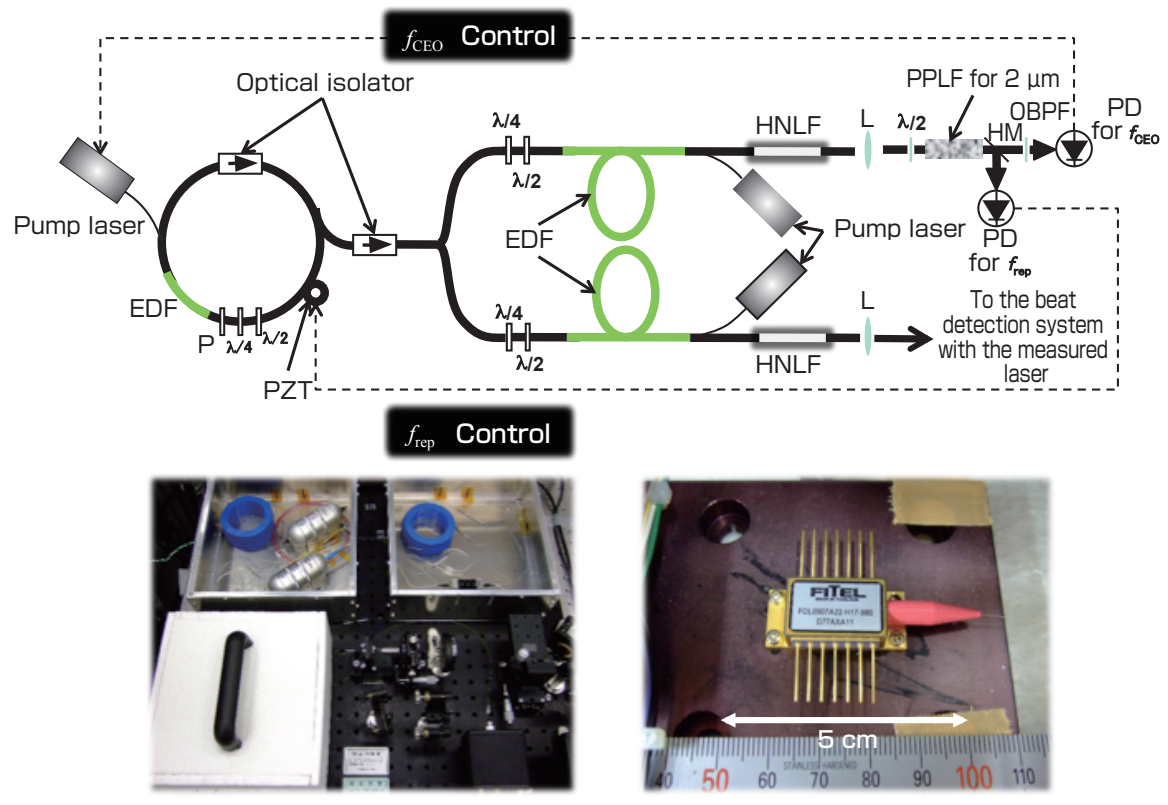

Fig. 3 Schematic view of the optical fiber comb system using the fiber laser

The fiber ring on the left is the mode-locked fiber laser that uses nonlinear polarization rotation as the mode-locking mechanism. The output is branched into two to four, each of which is amplified and/or broadened as required for use. All sections represent the optical fiber systems and enable continuous operation over a long period of time. The photos show the section between the laser and optical amplifier (right) and a pump laser (left) of the optical frequency comb system used as the national measurement standard. EDF: erbium-doped optical fiber; PZT: piezoelectric transducer; P: polarizer; $\lambda / 2$ : 1/2-wavelength plate; $\lambda / 4$ : 1/4-wavelength plate; HNLF: highly nonlinear fiber; L: lens; PPLN: periodically poled lithium niobate; HM: half-transparent mirror; OBPF: optical bandpass filter; PD: photo detector. 
lattice clocks," and other objectives as well as technologies involved in their development. Furthermore, we want to transfer these research outcomes to companies or universities in order to ultimately contribute to the improved reliability of time-frequency and length standards (Fig. 4).

When we began the development of the fiber comb before 2000, however, we were not certain that the fiber comb could achieve a performance equivalent to that of the Ti:S comb. We in fact needed to undertake some research steps to develop a fiber comb that could replace the Ti:S comb. This section describes the process from joint research with a company in the early stages of development of the optical frequency comb, to the transfer to the framework to fabricate the overall system within AIST, and to the establishment of the fabrication techniques.

\subsection{From offset beat detection to absolute frequency measurement - joint research with companies}

The mode-locked Ti:S laser and mode-locked fiber laser oscillate in the 800-nm band and in the 1550-nm band (the optical communication band), respectively. The power and optical pulse duration of the Ti:S laser are $300-800 \mathrm{~mW}$ and 10-30 fs, respectively, while those of the fiber laser are around $1-10 \mathrm{~mW}$ and $100 \mathrm{fs}$. It is necessary to find the conditions for broadening the spectra of those pulses with significantly different properties. The Ti:S laser uses a photonic crystal fiber with zero-dispersion wavelength around an 800-nm band for broadening, which cannot be directly applied to the fiber laser oscillating in the $1.5-\mu \mathrm{m}$ band. The company that engaged in joint research (19992004) provided AIST with a state-of-the-art and reliable mode-locked fiber lasers, winning the world's leading market share. We achieved the world's first frequency measurement using a mode-locked fiber laser, including a frequency link ${ }^{[9]}$ between the 778-nm and 1556-nm bands.

We further aimed to detect the $f_{\mathrm{CEO}}$ signal by broadening the second harmonic of this laser by means of the photonic crystal fiber. Although the use of a photonic crystal fiber does not even broaden the spectrum of the comb to less than an octave, we use a longer fiber for the Ti:S laser. We successfully observed the $f_{\text {CEO }}$ signal of the fiber comb for the first time in the world by using a new self-referencing method ${ }^{[10]}$ in which the optical frequency comb components broadened by the photonic crystal fiber interfered with the third harmonic of the laser in the 520-nm band. Shortly after, the octave-spanning optical frequency comb was achieved by the highly nonlinear fiber with a zero-dispersion wavelength in the $1.5-\mu \mathrm{m}$ band. We also developed the control and other systems in collaboration with the company and successfully performed an absolute frequency measurement of the stabilized laser using the fiber comb for the first time in the world. ${ }^{[1]}$

\subsection{From transfer to self-fabrication to long-term continuous operation}

Once absolute frequency measurement became feasible by means of the fiber comb, the consequent requirement was the ability to "customize specifications according to purposes or applications" or "provide multiple sets of equipment for different applications." To this end, we reached the conclusion that it would be quicker and easier to assemble parts on our own rather than receiving laser systems from a company. Fortunately being used in the major industrial area of optical communication, fiber and optical parts for the 1550-nm band are often both affordable and outstanding. Prior to our commitment to research into combs, we engaged

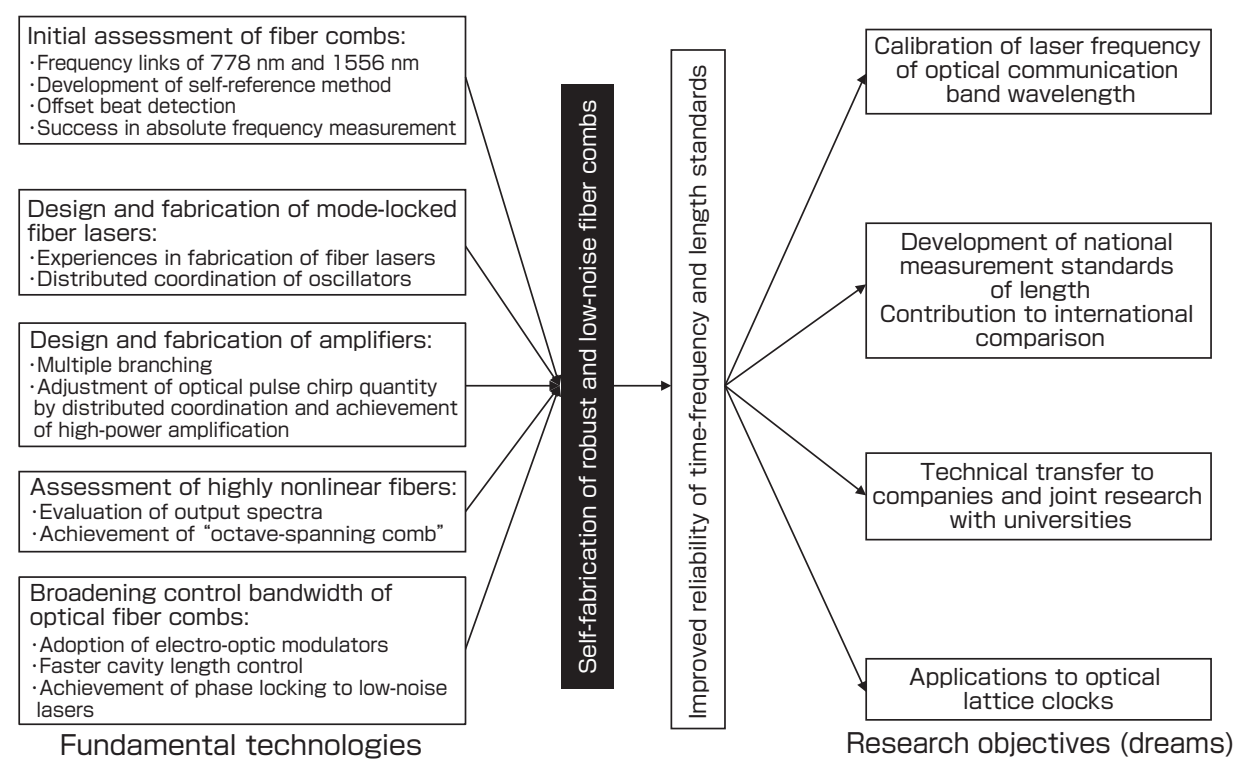

Fig. 4 Scenarios from fundamental technologies for self-fabrication of fiber combs to research objectives 
in $\mathrm{R} \& \mathrm{D}$ of a CW fiber laser, during which we obtained technical findings on the fiber optical system. This led us to the next objective of fabricating the fiber comb system inhouse (hereinafter referred to as self-fabrication).

Around the end of 2004 when we launched the selffabrication effort, we aimed to establish a simple and robust structure, eliminating any mechanisms unnecessary for the purposes or applications and facilitating fabrication, based on the abovementioned demands. For instance, the use of saturable absorbers or other special devices that were hardly available was avoided and the mode-locking mechanism was changed to a nonlinear polarization rotation to make up the structure, eliminating the space optical system to the maximum possible extent and focusing on fibers. However, the first attempt to fabricate a mode-locked laser on our own did not go well. During the same period, the highly nonlinear fiber with the best possible performance (even to this date) functioning in the $1-2 \mu \mathrm{m}$ range was available as a key to broaden the comb.

With this basis, we successfully designed and fabricated a mode-locked fiber laser and an ultrashort optical pulse amplifier, broadened the optical frequency comb by means of the highly nonlinear fiber, detected the offset frequency signal, arranged and fixed the fiber system to ensure robustness, and finally constructed the control system for phase lock or temperature control, as early as about a year from the beginning of the self-fabrication effort. In the beginning of 2006, we achieved optical frequency measurement $^{[12]}$ for a full consecutive week, a long-term measurement that had never previously been reported with the Ti:S comb or even with the fiber comb. This robustness has been accounted for using our unique laser systems, including the replacement of all components of the modelocked fiber laser by fibers, the branching structure (Fig. 3) of the optical amplifier section used to gain the $\mathrm{S} / \mathrm{N}$ ratio of a high beat signal, and the selection of the highly nonlinear fiber optimal for generating the optical frequency comb of the intended wavelength. These are the achievements of the self-fabrication effort.

Long-term continuous measurement soon brought about some practical findings. We have developed iodine-stabilized $\mathrm{Nd}$ :YAG lasers in the 532-nm band with high frequency stability and outstanding robustness. ${ }^{[13]}$ A gradual decrease in the frequency of one of the several lasers we kept was observed through long-term measurement (Fig. 5, top). The long-term continuous measurement using the fiber comb observed a reduction in frequency at the same pace (about $-20 \mathrm{~Hz}$ per week) (Fig. 5, bottom) and revealed that the frequency changes were not intermittent but continuous. ${ }^{[12]}$ This method of long-term continuous measurement allows us to observe phenomena we could not previously see. In the application to optical frequency standards that will be put to practical use, continuously measurable robustness is also one of the vitally required features.

\subsection{Importance of dispersion adjustment - establishing fabrication techniques}

Although the first self-fabricated fiber comb system was completed and was successful in continuous measurement, there were problems in the poor reproducibility of power or spectra from the ultrashort optical pulse amplifiers or in broadening by the highly nonlinear fiber in the subsequent processes of fabricating several mode-locked fiber lasers (oscillators) and ultrashort optical pulse amplifiers. Examining the polarization dependency or the mean and peak powers entering the optical pulse amplifier revealed that these problems were caused by a difference in the chirp quantities of optical pulses arising from dispersion of optical fibers linking the oscillators with the amplifiers.

While it was well known that dispersion adjustments of the oscillator, and of the optical path between the amplifier and highly nonlinear fiber were necessary, the importance of the dispersion adjustment of the optical path between the oscillator and amplifier was not well known. The results of past experiments only reported the chirped pulse amplification method, ${ }^{[14]}$ in which optical pulses were significantly chirped on either the positive or negative side before the amplifier in order to reduce their peak power for amplification, followed by chirping on the other side in order to compress the pulses. We determined the ideal length of optical fiber between the oscillator and amplifier required to maximize the mean power from the amplifier and to change the chirp quantity of optical pulses entering the amplifier (Figure 6). ${ }^{[15]}$ Under these conditions, optical pulses are amplified while being compressed by chirp compensation in order to obtain high optical pulse peak power, narrow
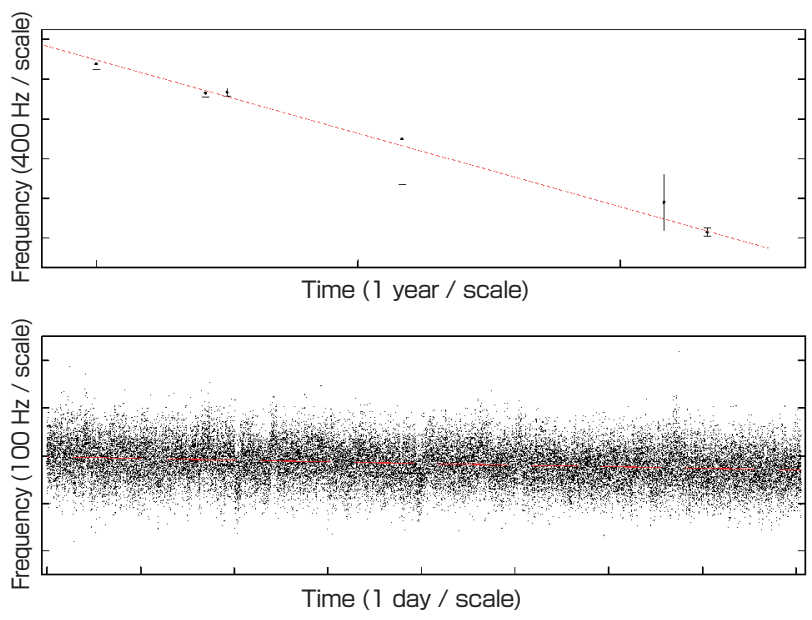

Fig. 5 Frequency changes of our iodine-stabilized Nd:YAG laser in the 532-nm band

Results of intermittent measurements with the Ti:S comb for 2.5 years (top) and a consecutive week of measurement with the fiber comb (bottom). Both results show a rate of change of about $-20 \mathrm{~Hz}$ per week. 
optical pulse duration, wide spectrum, and high average power. These are consistent with the conditions described as "adiabatic compression" in a report on broadened spectra in $1990{ }^{[16]}$ however, it was not known that they were associated with an increase in average power. The reason for this increased power remains unknown but it could be that a number of erbium ions with inhomogeneous broadening of gains can contribute to the optical pulse amplification, because the pulse spectrum is broadened, and thus resulting in high average power.

This study also revealed that the optical pulse amplified under these conditions is optimal for broadening the spectra by means of the highly nonlinear fiber and that they broaden more easily than optical pulses amplified under other conditions. This discovery significantly contributed to the improved reproducibility of power and spectra and established an important foundation for the subsequent "mass production" of fiber combs in laboratories. Furthermore, we applied for a patent on this method and equipment, which was then registered in January 2013 (Registration No.: 5182867).

\subsection{Development of a high-speed controllable frequency comb - evolution to a sophisticated and practical optical frequency comb}

The fiber comb was said to be practical and robust but had greater phase noise than the Ti:S comb, and an $f_{\text {CEO }}$ signal with a relatively broad linewidth unique to the fiber comb has been observed. ${ }^{[10]}$ While the spectral linewidth of $f_{\text {CEO }}$ is 100 $\mathrm{kHz}$ or less for the Ti:S laser in free operation, it could be as large as several megahertz for the fiber comb, which was for a while discussed as the most remarkable defect of the fiber comb. It has been revealed that phase noise derives not from

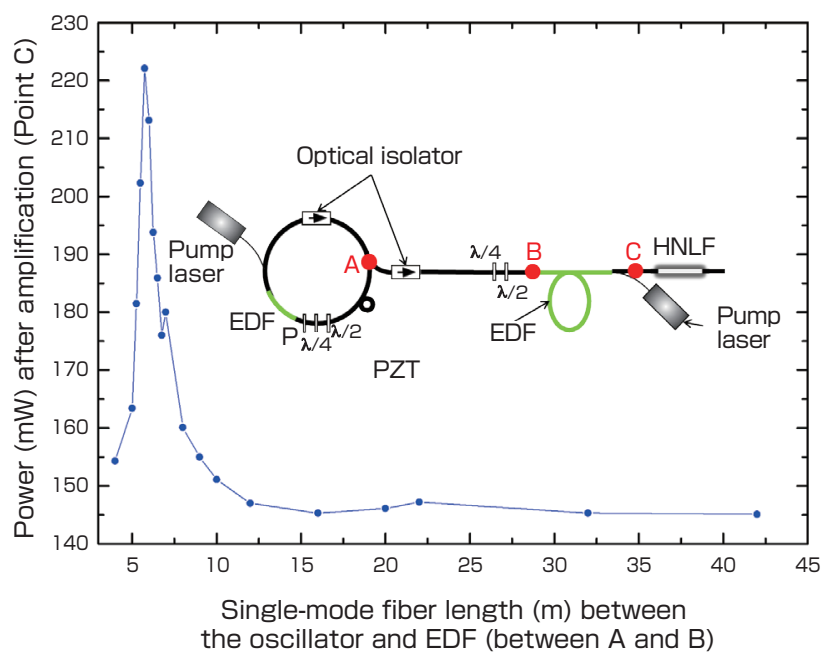

Fig. 6 Variation in average optical power at the optical amplifier section (Point $\mathrm{C}$ ) when varying the length of the single-mode fiber (SMF) between the oscillator output (Point $A$ ) and optical amplifier input sections (Point B)

The length of SMF needed to maximize average power is the optimum value for generating the octave-spanning optical frequency comb. the optical pulse amplifier or the highly nonlinear fiber but from the mode-locked fiber laser (oscillator). The subsequent accumulation of knowledge regarding oscillator fabrication, including total dispersion of the cavity, ${ }^{[17]}$ has elevated the fiber comb to an equivalent level to the Ti:S comb in terms of phase noise.

The most important aspect that not only completely solves the problems of phase noise but also makes the fiber comb exceed the capability of the Ti:S comb is high-speed controllability. The frequency values of the optical frequency comb have two degrees of freedom. For example, an optical frequency measurement needs to control $f_{\text {rep }}$ and $f_{\text {CEO }}$ independently. In most cases, $f_{\text {CEO }}$ is controlled by changing the pump power for the mode-locked laser while $f_{\text {rep }}$ is controlled by changing the cavity length. While pump power is controllable at a relatively high speed with the Ti:S or fiber laser, cavity length is hard to control at a high speed. These lasers generally change their cavity length by moving the mirror or fiber with the piezoelectric transducer. However, the servo bandwidth in this case is limited to several hundred to several tens of kilohertz. When $f_{\text {rep }}$ is phase-locked to the microwave frequency standard, the narrow servo bandwidth would be mostly unproblematic owing to the low carrier frequency and the small absolute quantity of the phase noise. Nevertheless, if one of the modes of the optical frequency comb is phaselocked to the optical frequency standard (a stabilized laser) by controlling cavity length, then the high carrier frequency results in a large absolute quantity of the phase noise, despite the same frequency stability, causing a shortage in gains and servo bandwidth of the cavity length control and difficulties in reducing phase noise. The relative spectral linewidth of the optical frequency comb will not be improved if much phase noise remains. The improvement of the relative spectral linewidth requires two parameters to be controlled at a high speed. We developed a high-speed controllable frequency comb with the electro-optic modulator (EOM) inserted into the cavity of the mode-locked fiber laser ${ }^{[18]}$ with which we achieved control to around the 1-MHz band for both lasers. ${ }^{[19]}$ The mode-locked laser with the intra-cavity EOM has only been possible for fiber laser, which has been an advantage of the fiber comb.

\subsection{Comparison with the conventional Ti:S comb or the fiber comb using Yb-added optical fibers}

By employing the mode-locked fiber laser as the light source of the optical frequency comb, a number of defects in the conventional, mainstream Ti:S comb have been overcome through the developments stated in this section. The fiber comb that we have developed uses erbium (Er)-doped optical fiber with gains in the $1.5-\mu \mathrm{m}$ band as the laser medium, while some other groups have developed fiber combs using ytterbium (Yb)-doped optical fiber with gains in the $1-\mu \mathrm{m}$ band. Table 1 shows a list of features of the Ti:S comb, Er fiber comb, and $\mathrm{Yb}$ fiber comb. 
Table 1. Characteristics of optical fiber combs depending on the types of mode-locked lasers

\begin{tabular}{|l|l|l|l|}
\hline & Ti:S comb & Er fiber comb & Yb fiber comb \\
\hline Output (mean power) & Up to $1 \mathrm{~W}$ & $\begin{array}{l}\text { Up to } 200 \mathrm{~mW} \\
\text { (after amplification) }\end{array}$ & $\begin{array}{l}\text { Up to } 10 \mathrm{~W} \\
\text { (after amplification) }\end{array}$ \\
\hline $\begin{array}{l}\text { Optical pulse width } \\
\text { (typical value after } \\
\text { chirp compensation) }\end{array}$ & $\begin{array}{l}\text { Several } \\
\text { femtoseconds }\end{array}$ & $\begin{array}{l}\text { Several tens of } \\
\text { femtoseconds }\end{array}$ & $\begin{array}{l}\text { Several tens of } \\
\text { femtoseconds }\end{array}$ \\
\hline $\begin{array}{l}\text { Wavelength } \\
\text { (typical central } \\
\text { wavelength of an } \\
\text { oscillator) }\end{array}$ & $780 \mathrm{~nm}$ & $1550 \mathrm{~nm}$ & $1030 \mathrm{~nm}$ \\
\hline $\begin{array}{l}\text { Wavelength range } \\
\text { (typical value after } \\
\text { broadening) }\end{array}$ & $400-1200 \mathrm{~nm}$ & $900-2500 \mathrm{~nm}$ & $\begin{array}{l}900-2500 \mathrm{~nm} \\
\text { or } \\
700-1400 \mathrm{~nm}\end{array}$ \\
\hline $\begin{array}{l}\text { Spectral linewidth } \\
\text { of the CEO signal } \\
\text { in free operation }\end{array}$ & $\begin{array}{l}\text { Several tens of } \\
\text { kHz to several MHz }\end{array}$ & $\begin{array}{l}\text { Several tens of } \\
\text { kHz to several MHz }\end{array}$ & $\begin{array}{l}\text { Several tens of } \\
\text { kHz to several MHz }\end{array}$ \\
\hline $\begin{array}{l}\text { Bandwidth control } \\
\text { (cavity length) }\end{array}$ & $\begin{array}{l}\text { Up to several tens } \\
\text { of KHz }\end{array}$ & Up to several MHz & Up to several MHz \\
\hline Pump laser & $\begin{array}{l}\text { Mainly solid-state } \\
\text { laser }\end{array}$ & $\begin{array}{l}\text { Mainly } \\
\text { semiconductor laser }\end{array}$ & $\begin{array}{l}\text { Mainly } \\
\text { semiconductor laser }\end{array}$ \\
\hline $\begin{array}{l}\text { Difficulty of } \\
\text { long-term operation }\end{array}$ & $\begin{array}{l}\text { Difficult (instability } \\
\text { of free space } \\
\text { optical system and } \\
\text { pump laser) }\end{array}$ & Easy & $\begin{array}{l}\text { Easy but free-space } \\
\text { optical system is } \\
\text { required for the } \\
\text { cavity-dispersion } \\
\text { compensation }\end{array}$ \\
\hline
\end{tabular}

A comparison of these three types of optical frequency comb shows that our Er fiber comb has advantages not for the performances of optical pulses in the time domain, including power and pulse width, but for the performances of the optical frequency comb in the frequency domain, like low noise (narrow spectral linewidth), reliability, and high-speed control.

As the Ti:S comb and $\mathrm{Yb}$ fiber comb have such advantages as high power, short wavelength, or narrow optical pulse width, these optical frequency combs would be the only solution to a number of applications in the ultraviolet region or those requiring high resolution in the time domain. However, as far as frequency metrology with a wavelength longer than the visible region is concerned, the Er fiber comb is the most sophisticated and practical.

\section{Expanded fiber comb applications}

The self-fabrication of fiber combs enabled us to select robust, easy-to-use, and sophisticated optical frequency comb systems according to the intended purposes. As was expected in the beginning, it was an inevitable result of developing optical frequency combs in research or business applications. Our group has already completed more than fifteen fiber comb systems. This section provides particularly important explanations of several topics, including the "calibration of the optical communication band wavelength laser," "national measurement standards," and "development of the highspeed controllable frequency comb."

\subsection{Frequency calibration of lasers in the optical communication band}

The demand for increased transmission capacity in optical communication has been steady growing. Multiplexing the communication bandwidth and increasing the number of channels is effective for a greater transmission capacity. However, frequency management is required to set the communication channels, owing to the limited power to transmit through the single-mode fibers and to the limited range of wavelengths that are low in transmission loss. As a platform for this wavelength division multiplexing, the frequency grid (ITU-T G694.1) is available at $12.5 \mathrm{GHz}$, $25 \mathrm{GHz}, 50 \mathrm{GHz}$, and $100 \mathrm{GHz}$ intervals from $193.1 \mathrm{THz}$, which is the carrier frequency of the communication band C. The recent rapid development of digital coherence techniques (digital signal processing that has been used in the field of radio, applied to optical communication) has led to wavelength multiplexing up to near the theoretical limit of the transmission capacity of a single-mode fiber. For an application to the flexible grid (a channel at 6.25$\mathrm{GHz}$ intervals), a reduction of uncertainty in the frequency management technology, including frequency measurement, would be increasingly important.

When manufacturers of optical measuring instruments or optical devices require 7 to 8 -digit precision for optical spectrum analyzers or wavemeters, a laser stabilized to an absorption line of a molecule is used, which provides around 9-digit precision. We began developing the wavelength standards in the 1.5- $\mu \mathrm{m}$ band, foreseeing these needs before the optical frequency comb had become available. Our acetylene-stabilized laser with a $1542-\mathrm{nm}$ wavelength ${ }^{[20]}$ has been recommended as the only wavelength standard in the $1.5-\mu \mathrm{m}$ band by the CIPM. Furthermore, laser frequency calibration in the $1510-1570-\mathrm{mm}$ band became feasible by broadening the power of this laser by means of the sideband optical frequency comb. ${ }^{[21]}$ Problems, however, still remained. First, calibrations by the stabilized laser and the side-band optical frequency comb required the international comparison of the stabilized laser itself as the reference frequency or calibration with the optical frequency comb. The national measurement standards for wavelength were the iodine-stabilized He-Ne laser with a wavelength of $633 \mathrm{~nm}$, which could fall into a double standard. On the other hand, the optical frequency comb measures frequency (wavelength) based on the frequency standards and allows the wavelength in both the $633-\mathrm{nm}$ and $1.5-\mu \mathrm{m}$ bands to directly connect to the International System of Units (SI) without the use of the stabilized laser. It also facilitates the actions to be taken when traceability needs to be secured with different wavelengths in the future. Having said that, the initial Ti:S comb could broaden the spectral range by only $500-1100 \mathrm{~nm}$ and is not very applicable to the optical communication band wavelength. This is because the measured laser with $\mathrm{CW}$ light is less efficient in generating the second harmonic.

The fiber comb operates in the $1-2-\mu \mathrm{m}$ band and covers all optical communication band wavelengths. Manufacturing such components as light sources or optical filters to cover further downsized and complicated communication grids 
requires wavemeters or optical spectrum analyzers of higher precision and, as the reference standards of these measuring instruments, stabilized lasers with different wavelengths in the optical communication band will be required. This bandwidth range contains a number of transitions preferred as wavelength standards for acetylene molecules or hydrogen cyanide molecules, which seems to indicate that the fiber comb is optimal for calibrating stabilized lasers of those transitions. In addition, the optical frequency comb uses optical pulses that are highly efficient in second-harmonic generation and is also applicable to the visible wavelength range. It is thus expected to replace the Ti:S comb.

\subsection{National measurement standards of length}

The traceability system for both wavelengths $(633 \mathrm{~nm}$ and $1.5 \mu \mathrm{m}$ ) can be simplified if we set the atomic clock (frequency standard) and an optical frequency comb as the national measurement standards of length (Fig. 7), which can also achieve length standards faithful to the definition of the meter. The iodine-stabilized He-Ne laser with a 633$\mathrm{nm}$ wavelength, which had been the conventional national measurement standard, is compact, has an uncertainty as low as $2.5 \times 10^{-11}$, and is complete as a length standard. However, some issues remain in using it as the national measurement standard. One is that, as a stabilized laser, it requires periodic calibration using the optical frequency comb or international comparison. Considering the possible fluctuations of frequency values after cavity alignment, group management of several equivalent units must also be carried out to ensure that each unit runs properly.

The secondary standards (i.e., devices directly calibrated by the national measurement standards) owned by accredited calibration laboratories are also iodine-stabilized $\mathrm{He}-\mathrm{Ne}$ lasers with no difference in performance from the national measurement standards, which means that calibration results are not necessarily successful in properly evaluating the performances of the measured object. If the national measurement standards have the same uncertainty as the secondary standards, then the uncertainty of calibration results will be $\sqrt{2}$ times as much as each uncertainty. This means the uncertainty assumed to be equal to that of the national measurement standards would be underestimated.

To avoid such degradation, the uncertainty was regarded as being the same as that of the national measurement standards, assuming the secondary standards to be equivalent to the national measurement standards if the compared frequencies were found to be within the normal range. A comparison between iodine-stabilized He-Ne lasers employed a method of estimating the original frequency difference by measurement of a total of six differential frequencies by locking each laser at four absorption lines (a matrix method) as no measurement could be made where there was too small a difference in frequency. Calibration therefore required a great deal of time and effort. Furthermore, the iodinestabilized He-Ne laser is sensitive to vibration and noise. The unit at AIST is reinforced against such vibration or noise but is still somewhat sensitive.

Our fiber comb solves all these problems. The reference frequency of the optical frequency comb represents the frequency standard in synchronization with the International Atomic Time (TAI), requiring no periodic calibration. Essentially, a defect in microwave frequency synthesis (included in the " $f_{\text {rep }}$ control" in Fig. 3 ) would be the only possible cause for values to deviate, but this can also be detected during measurement, and therefore requires no group management. With an uncertainty $1 / 300$ th of the secondary standards, the calibration results almost completely show the performances of the device under test (DUT). This enables the frequency and uncertainty of calibration to be clearly provided in the calibration certificate. Also robust when locking, our fiber comb can facilitate operation for around one consecutive week, and calibration of the DUT only requires measurement of the frequency of the

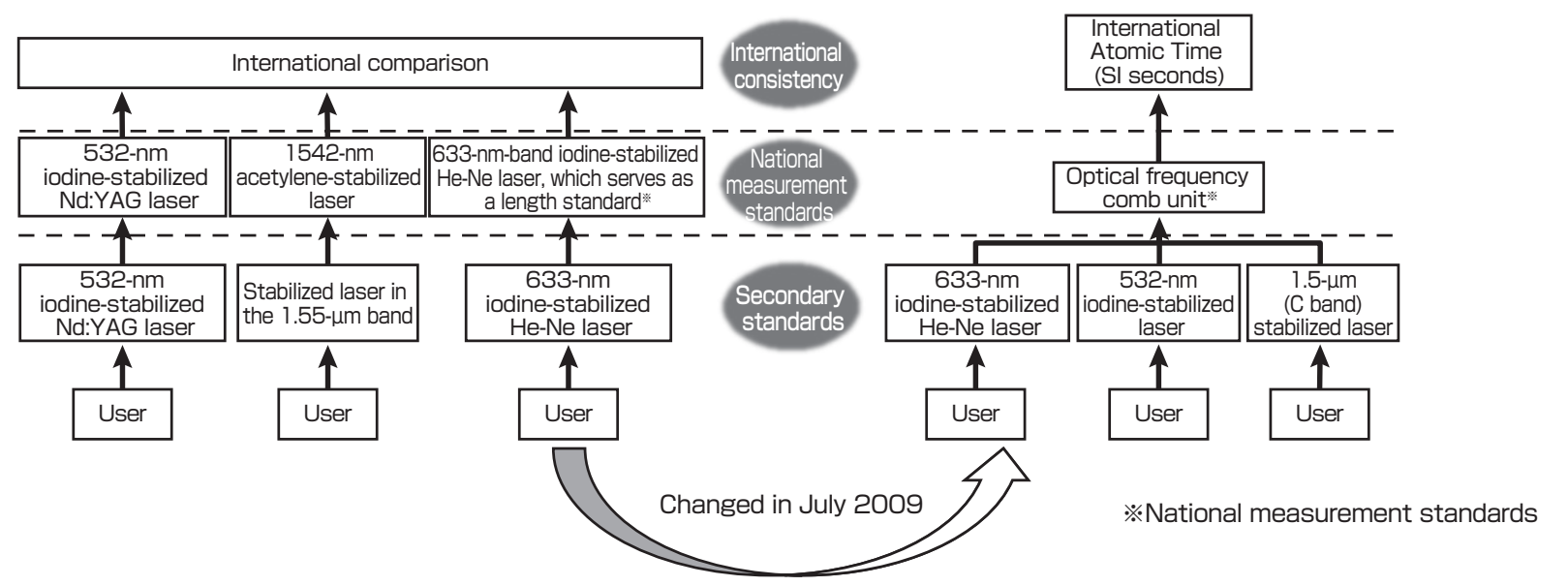

Fig. 7 Old and new traceability systems for length measurement (SI to secondary standards)

A simple traceability system has been achieved in all wavelength regions by setting the optical frequency comb as the national measurement standard. 
laser locked at the appropriate absorption line, resulting in a significant reduction in time and effort. The calibration work that once took a few days is expected to be completed in half a day to a day since the change in the national measurement standards in July 2009.

\subsection{International comparison of the frequency- stabilized laser}

It is also important to verify international equivalence. National metrology institutes from different nations must gather their stabilized lasers for international comparison in order to verify their equivalence. In the $20^{\text {th }}$ century, researchers from the International Bureau of Weights and Measures brought their portable iodine-stabilized He-Ne laser with them to different regions, where the frequency of their laser was compared with iodine-stabilized He-Ne lasers from other regions or nations. This is how the equivalence of iodine-stabilized He-Ne lasers around the world had formerly been verified.

However, a review of works by the International Bureau of Weights and Measures led to the launch of a new international comparison system (CCL-K11) for the stabilized laser in 2004, under which an international comparison came to be carried out by each grouping of national metrology institutes in each region. With a pilot laboratory to begin with, a "node laboratory" that leads the relevant region is set up for nine large or small groupings of national metrology institutes in different regions, including the Asia Pacific Metrology Programme (APMP). The node laboratory measures the frequency of the frequency-stabilized laser using the optical frequency comb on a periodic basis. The national metrology institutes from different nations bring their lasers to the node laboratory of the region to measure frequency and verify equivalence. The equivalence of frequency measurements between different node laboratories is ensured by the Coordinated Universal Time (UTC) maintained by node laboratories and Calibration and Measurement Capability (CMC) registration of optical frequency combs.

Of the nations in the APMP, Japan, China, Korea, Singapore, and Australia owned their own optical frequency combs, some nations of which also owned fiber combs as of 2010. However, while those nations, except Japan, purchased units that were commercially available, AIST of Japan engaged in self-fabrication. AIST has been recognized for their related technologies and has been designated as the node laboratory in the APMP region. The international comparison of the iodine-stabilized He-Ne laser that was carried out at AIST in April 2010 was a symbolic event that demonstrated the superiority of our fiber comb. The national metrology institutes of eight nations in the Asian and Oceanian regions brought their 633-nm iodine-stabilized He-Ne lasers to AIST, the node laboratory, to compare frequencies of the lasers using the fiber comb we have developed.
Using our robust and accurate fiber comb to simplify measurements as described above, the measurement went extremely well and was fully completed in around a day. Moreover, a time-consuming long-term frequency stability measurement was successfully made upon request from the institutes. This helped evaluate the performances of all stabilized lasers, with no anxiety of unmanned operation of fiber combs even at midnight. The results of laser measurements from the eight nations were within the uncertainty range in a list of recommended values of standard frequencies by the General Conference on Weights and Measures, indicating the equivalence of the stabilized lasers. ${ }^{[22]}$ These lasers now play a role as the national measurement standards in their nations. This means that traceability of the SI length in these eight nations always passes through AIST fiber comb.

\subsection{Application of the high-speed controllable frequency comb to the optical lattice clock}

Our group has developed the "optical lattice clock",[23] to be the next-generation frequency standard. The "optical clock" represented by the optical lattice clock, based on the clock transition on the optical frequency domain, requires a narrow spectral linewidth at the hertz level of the laser for observing clock transitions, owing to the extremely narrow spectral linewidth of the transition and the low probability of transition. The method employed to achieve such a laser regulates the temperature of a cavity that has high finesse and a low rate of thermal expansion in vacuum for high stabilization, and stabilizes the laser to a longitudinal mode of the cavity. We have developed $\mathrm{Yb}$ and Sr optical lattice clocks, with clock transition wavelengths of $578 \mathrm{~nm}$ and $698 \mathrm{~nm}$, respectively. While ultrastable cavities are generally used for each clock transition wavelength, we use an ultrastable cavity for $1064 \mathrm{~nm}$, different from the clock transition wavelengths. This is in order to achieve "linewidth transfer" (Fig. 8), where the frequency stability and spectral linewidth of the 1064-nm cavity are transferred to the 578$\mathrm{nm}$ and 698-nm cavities using the high-speed controllable optical frequency comb. ${ }^{[24]}$

The advantages of this method include the following points. (1) A reliable and stable laser system can be realized because a cavity can be used for wavelengths available with a robust and sophisticated laser (e.g., $1064 \mathrm{~nm}$ ), and only a single ultrastable cavity is required. (2) Frequency variations in the reference-stabilized laser can be compensated by measuring the frequency ratio of the two optical lattice clocks, which consequently offers a high frequency stability that no other method can achieve. (3) The mode spacing of the optical frequency $(40-200 \mathrm{MHz})$ is smaller than the free spectral range of the ultrastable cavity (around $2 \mathrm{GHz}$ in general), thus increasing the range of options in selecting an acoustooptic modulator to be used as a bridge to the clock transition frequency. This method will be an important technology in 
operating several optical clock systems.

\section{Conclusion}

How far a unit should be disassembled for self-fabrication or whether it is simpler to purchase a commercially available item is a difficult issue. Fortunately, however, our attempt to self-fabricate a mode-locked fiber laser and ultrashort optical pulse amplifier was more successful than anticipated. It enabled optimization of lasers and amplifiers for design and fabrication and enabled quick changes in specifications to help take advantage of the design and development of optical systems, laser control, frequency measurement, and other technologies that we have proved capable of, which dramatically accelerates the development speed. As a result, the optical frequency measuring instrument once available for use only for a short period of time, and the optical frequency comb formerly only a tool for demonstration, have been adapted to calibration of optical communication band wavelengths. They have not only successfully replaced national measurement standards for length but have also been successfully applied to the laser systems of optical lattice clocks and in other practical contexts. One may argue that this is the second breakthrough required to put the optical frequency comb in practical use.

With respect to the national measurement standards, we have long realized the importance of calibration services in the optical communication band and have always taken initiative in the establishment of traceability systems and calibration works. The recent steady increase in the number of tests requested demonstrates the strategic significance of performing optical measurement in the optical communication band. The completion of a largescale international comparison with as many as eight nations within a short time was something we did not anticipate but it served as a symbolic example demonstrating the superiority of the fiber comb.

Although it has high reliability and a relatively simple configuration, the fiber comb needs improved reliability at a higher level and an even simpler configuration when taking into account commercialization or applications to other fields. To this end, we need to collaborate with companies. We have experience in technical transfer through joint research or technical training with several companies taking advantage of programs, including the "Grant for Industrial Technology Research" program of the New Energy and Industrial Technology Development Organization. We also aim to commercialize sophisticated fiber combs by dividing fiber optical systems and control systems for modularization. If the fiber comb is downsized, commercialized, and offered at lower prices, then it could possibly be used not only for calibration but also as communication technology itself, including direct generation of signal light strictly conforming to the grid wavelength in the area of optical communication. Furthermore, in the area of length standards, accredited calibration laboratories will own their own optical frequency combs, ultimately eliminating the necessity of transporting iodine-stabilized He-Ne lasers. Optical communication technologies, traceability systems in Japan, and the CCL-K11 international comparison system for stabilized lasers are expected to evolve into more reasonable dimensions as time goes on, and we expect our fiber comb to be of some help in this evolution.

\section{Terminologies}

Term 1. Mode-locked laser: If loss or modulation of the index of refraction is applied to a cavity at a frequency close to the free spectral range, then the free spectral range will be drawn into and

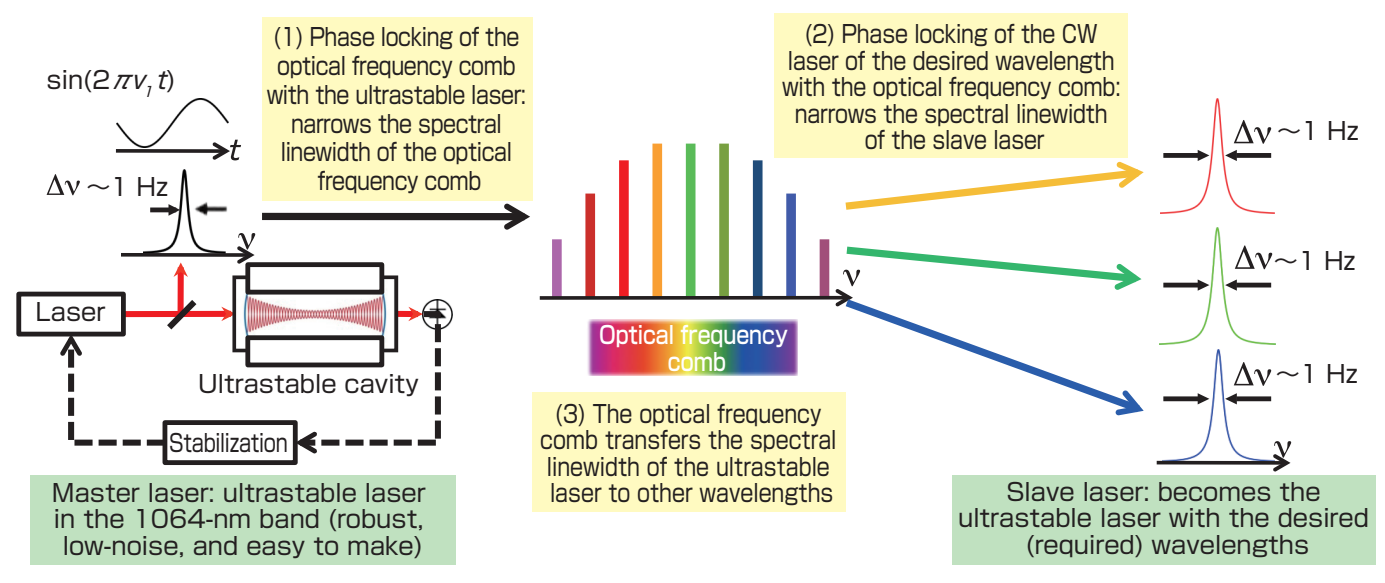

Fig. 8 "Linewidth transfer" using the optical frequency comb

The spectral linewidths of all modes for the octave-spanning optical frequency comb are narrowed down by phase-locking one of the modes for the optical frequency comb with a master laser for narrowing the spectral linewidth of a given wavelength, and at the same time by narrowing down the spectral linewidth of $f_{\mathrm{CEO}}$ by phase-locking with a broad servo bandwidth. This method provides the spectral linewidth and frequency stability of the master laser to the slave lasers in desired wavelengths. It also requires the "high-speed controllable frequency comb" that enables step (1) "Phase locking of the optical frequency comb with the ultrastable laser." 
equal to the modulation frequency. This is called mode locking. Mode locking by externally adding modulation is called active mode locking, while synchronization by fluctuations in light power in the cavity without adding external modulation is called passive mode locking.

Term 2. Highly nonlinear fiber: An optical fiber with a high nonlinear coefficient used for broadening the output spectrum of the mode-locked laser to an octave or more. It generally refers to a fiber with a zerodispersion wavelength in the $1.5-\mu \mathrm{m}$ band, though a photonic crystal fiber with a zero-dispersion wavelength around the $800-\mathrm{nm}$ band is also a highly nonlinear fiber.

Term 3. Side-band optical frequency comb: A type of optical frequency comb used before the optical frequency comb with the mode-locked laser was invented. It can acquire a number of relatively broadened side bands entered into the $\mathrm{CW}$ laser by inserting the electro-optic modulator into a cavity and applying a modulation frequency close to the free spectral range frequency. Although it yields an extremely high mode spacing frequency and high power per mode, no report has yet been made that obtained the CEO signal. The side-band optical frequency comb has been little used for frequency measurement after it was replaced by the optical frequency comb using the mode-locked laser.

Term 4. Asia Pacific Metrology Programme (APMP): Launched in 1980, the APMP conducts international comparisons of standards from different nations and technical cooperation as a grouping of national metrology institutes engaged in metrology activities based on the Metre Convention under the umbrella of Asia-Pacific Economic Cooperation (APEC). Apart from APMP, the SIM in North America, the EUROMET mainly located in the European continent, and six other large and small groupings of national metrology institutes around the world are in mutual cooperation.

Term 5. Calibration and Measurement Capability (CMC): Uncertainty of measurement of national measurement standards internationally accepted by examinations under the Metre Convention.

Term 6. Optical lattice clock: Compared to other "optical clocks" with the clock transition frequency in the optical region, the optical lattice clock can achieve both high accuracy and frequency stability. It is thought to be the best candidate for a next-generation frequency standard. Proposed by Dr. Katori, a professor at the University of Tokyo, this standard has been subject to research and development at leading national metrology institutes around the world over 10 years.

\section{References}

[1] H. Schnatz, B. Lipphardt, J. Helmcke, F. Riehle and G. Zinner: First phase-coherent frequency measurement of visible radiation, Phys. Rev. Lett., 76 (1), 18-21 (1996).

[2] Y. Miki, A. Onae, T. Kurosawa, Y. Akimoto and E. Sakuma: Frequency chain to $3.39-\mu \mathrm{m} \mathrm{CH}_{4}$-stabilized He-Ne-laser using Josephson point-contact as harmonic mixer, Jpn. J. Appl. Phys. Part 1, 33, 1655-1658 (1994).

[3] T. J. Quinn: Practical realization of the definition of the metre, including recommended radiations of other optical frequency standards (2001), Metrologia, 40 (2), 103-133 (2003).

[4] J. Ishikawa: Portable national length standards designed and constructed using commercially available parts, Synthesiology, 2 (4), 276-287 (2009) (in Japanese) [Synthesiology English edition, 2 (4), 246-257 (2010)].

[5] T. Udem, J. Reichert, R. Holzwarth and T. W. Hänsch: Absolute optical frequency measurement of the cesium $\mathrm{D}_{1}$ line with a mode-locked laser, Phys. Rev. Lett., 82, 35683571 (1999).

[6] D. J. Jones, S. A. Diddams, J. K. Ranka, A. Stentz, R. S. Windeler, J. L. Hall and S. T. Cundiff: Carrier-envelope phase control of femtosecond mode-locked lasers and direct optical frequency synthesis, Science, 288 (5466), 635-639 (2000).

[7] T. Okuno, M. Onishi, T. Kashiwada, S. Ishikawa and M. Nishimura: Silica-based functional fibers with enhanced nonlinearity and their applications, IEEE J. Sel. Top. Quant. Electron., 5, 1385-1391 (1999).

[8] J. K. Ranka, R. S. Windeler and A. J. Stentz: Optical properties of high-delta air silica microstructure optical fibers, Opt. Lett., 25, 796-798 (2000).

[9] A. Onae, T. Ikegami, K. Sugiyama, FL. Hong, K. Minoshima, H. Matsumoto, K. Nakagawa, M. Yoshida and S. Harada: Optical frequency link between an acetylene stabilized laser at $1542 \mathrm{~nm}$ and an Rb stabilized laser at $778 \mathrm{~nm}$ using a two-color mode-locked fiber laser, Opt. Commun., 183, 181-187 (2000).

[10] FL. Hong, K. Minoshima, A. Onae, H. Inaba, H. Takada, A. Hirai, H. Matsumoto, T. Sugiura and M. Yoshida: Broadspectrum frequency comb generation and carrier-envelope offset frequency measurement by second-harmonic generation of a mode-locked fiber laser, Opt. Lett., 28 (17), 1516-1518 (2003).

[11] T. R. Schibli, K. Minoshima, FL. Hong, H. Inaba, A. Onae, H. Matsumoto, I. Hartl and M. E. Fermann: Frequency metrology with a turnkey all-fiber system, Opt. Lett., 29 (21), 2467-2469 (2004).

[12] H. Inaba, Y. Daimon, FL. Hong, A. Onae, K. Minoshima, T. R. Schibli, H. Matsumoto, M. Hirano, T. Okuno, M. Onishi and M. Nakazawa: Long-term measurement of optical frequencies using a simple, robust and low-noise fiber based frequency comb, Opt. Express, 14 (12), 5223-5231 (2006).

[13] FL. Hong, J. Ishikawa, ZY. Bi, J. Zhang, K. Seta, A. Onae, J. Yoda and H. Matsumoto: Portable I2-stabilized Nd : YAG laser for international comparisons, IEEE T. Instrum. Meas., 50 (1), 486-489 (2001).

[14] D. Strickland and G. Mourou: Compression of amplified chirped optical pulses, Opt. Commun., 56 (3), 219-221 (1985).

[15] Y. Nakajima, H. Inaba, FL. Hong, A. Onae, K. Minoshima, T. Kobayashi, M. Nakazawa and H. Matsumoto: Optimized amplification of femtosecond optical pulses by dispersion management for octave-spanning optical frequency comb generation, Opt. Commun., 281 (17), 4484-4487 (2008). 
[16] M. Nakazawa, K. Kurokawa, H. Kubota, K. Suzuki and Y. Kimura: Femtosecond erbium-doped optical fiber amplifier, Appl. Phys. Lett., 57, 653-655 (1990).

[17] L. Nugent-Glandorf, T. A. Johnson, Y. Kobayashi and S. A. Diddams: Impact of dispersion on amplitude and frequency noise in a Yb-fiber laser comb, Opt. Lett., 36 (9), 1578-1580 (2011).

[18] Y. Nakajima, H. Inaba, K. Hosaka, K. Minoshima, A. Onae, M. Yasuda, T. Kohno, S. Kawato, T. Kobayashi, T. Katsuyama and FL. Hong: A multi-branch, fiber-based frequency comb with millihertz-level relative linewidths using an intra-cavity electro-optic modulator, Opt. Express, 18 (2), 1667-1676 (2010).

[19] K. Iwakuni, H. Inaba, Y. Nakajima, T. Kobayashi, K. Hosaka, A. Onae and FL. Hong: Narrow linewidth comb realized with a mode-locked fiber laser using an intra-cavity waveguide electro-optic modulator for high-speed control, Opt. Express, 20 (13), 13769-13776 (2012).

[20] A. Onae, K. Minoshima, J. Yoda, K. Nakagawa, A. Yamaguchi, M. Kourogi, K. Imai and B. Widiyatomoko: Toward an accurate frequency standard at $1.5 \mu \mathrm{m}$ based on the acetylene overtone band transition, IEEE T. Instrum. Meas., 48, 563-566 (1999).

[21] M. Kourogi, K. Nakagawa and M. Ohtsu: Wide-span optical frequency comb generator for accurate optical frequency difference measurement, IEEE J. Quant.Electron., 29, 26932701 (1993).

[22] M. Matus, P. Balling, P. Kren, P. Mašika, FL. Hong, J. Ishikawa, H. Inaba, K. Hosaka, M. Yasuda, D. Akamatsu, A. Onae, RH. Shi, JL. Peng, E. Howick, J. Qian, XY. Liu, M. Ranusawud, A. Tonmueanwai, S. L. Tan, ZX. Chao, KH. Yeoh, N. Mat Daud, T. K. Chan, R. Fira, O. Stalder, K. Tomanyiczka and L. Robertsson: The CCL-K11 ongoing key comparison: final report for the year 2010, Metrologia, 48 (2011).

[23] M. Yasuda, H. Inaba, T. Kohno, T. Tanabe, Y. Nakajima, K. Hosaka, D. Akamatsu, A. Onae, T. Suzuyama, M. Amemiya and FL. Hong: Improved absolute frequency measurement of the $171 \mathrm{Yb}$ optical lattice clock towards a candidate for the redefinition of the second, Appl. Phys. Express, 5 (2012).

[24] H. Inaba, K. Hosaka, M. Yasuda, Y. Nakajima, K. Iwakuni, D. Akamatsu, S. Okubo, T. Kohno, A. Onae and FL. Hong: Spectroscopy of $171 \mathrm{Yb}$ in an optical lattice based on laser linewidth transfer using a narrow linewidth frequency comb, Opt. Express, 21 (7), $7891-7896$ (2013).

\section{Authors}

\section{Hajime INABA}

Earned a Master's degree in applied physics, Graduate School of Engineering, Hokkaido University, in 1993. Joined the National Research Laboratory of Metrology the same year. Engaged in the development of continuous wave (CW) fiber lasers. Chief scientist, National Metrology Institute of Japan, National Institute of Advanced Industrial

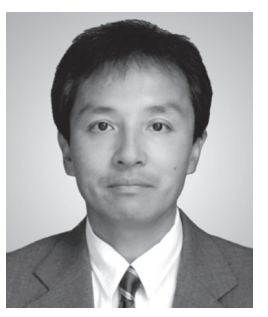

Science and Technology since 2001. Doctor of engineering at Hokkaido University in 2004. Engages in research into generation and control of the optical frequency comb and applications to technologies related to frequency standards. Received the Award of the Minister of Education, Culture, Sports, Science and Technology in 2008, and the Ichimura
Academic Award in 2012. In this study, he played a leading role in the self-fabrication of fiber combs, establishing national measurement standards, and achieving a narrower spectral linewidth.

\section{Atsushi Onae}

Dropped out of the doctoral program in Physics, Graduate School of Science, the University of Tokyo in 1988. Joined the National Research Laboratory of Metrology the same year. Doctor of Science at the University of Tokyo in 1990. Engaged in the development of wavelength standards in the optical communication band using acetylene molecules and

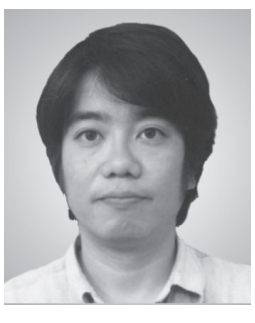
research into optical frequency measurement technology to evaluate the standards. Currently the senior chief scientist of the National Metrology Institute of Japan, National Institute of Advanced Industrial Science and Technology. Received the Japan Society of Applied Physics Outstanding Achievement Award in Optics and Quantum Electronics in 2003, and the Award of the Minister of Education, Culture, Sports, Science and Technology in 2008. In this study, he played a leading role in technical development in the optical communication band wavelength (the development of stabilized lasers and frequency measurement using the optical frequency comb).

\section{Feng-Lei HoNG}

Completed the doctoral program in Physics, Graduate School of Science, the University of Tokyo in 1992. Special Postdoctoral Research, RIKEN from 1992. Joined the National Research Laboratory of Metrology in 1994, and is currently the Director of the Time and Frequency Division, National Metrology Institute of Japan, National Institute of Advanced

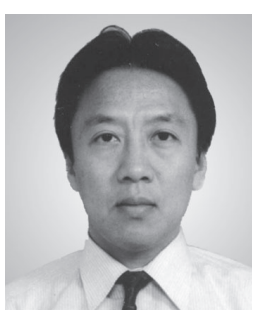
Industrial Science and Technology. Engages in research into high-resolution laser spectroscopy, optical frequency combs, and optical frequency measurement. Received the Award of the Minister of Education, Culture, Sports, Science and Technology in 2008, and the Ichimura Academic Award in 2012. In this study, he played a leading role in achieving the key developments in fiber combs during the initial stages, the establishment of national measurement standards for fiber combs, and international comparisons of stabilized lasers.

\section{Discussions with Reviewers}

\section{Overall}

Comment (Naoto Kobayashi: Center of Research Strategy, Waseda University)

I would say that this paper on optical frequency comb generation is an excellent work suitable for Synthesiology in the sense that it overcame a number of drawbacks of conventional comb generation with the solid-state laser by means of the fiber comb, and demonstrated outstanding performance in relation to standards in the optical communication frequency domain.

Comment (Hidemi Tsuchida: AIST)

This paper is regarded as research and development for establishing an optical frequency comb apparatus for practical purpose, based on the invention of the fundamentals by Hall and Hänsch. Regarding the development of the fiber-based optical comb, the paper clearly describes the target and the approach leading to the goal. 


\section{Title}

Question and comment (Hidemi Tsuchida)

While you have entitled the paper as "National standards of length for high-capacity optical fiber communication systems," the requirement of the optical communication systems is not "length" but "optical frequency" standards. Is it possible to change the title so as to fit the intention of the paper, for example, into "National standards of optical frequency for high-capacity optical fiber communication systems"?

\section{Answer (Hajime Inaba, Atsushi Onae, and Feng-Lei Hong)}

Support for the communication band is obviously an important mission, and we have constantly taken initiatives in establishing calibration technology. On the other hand, however, developing the technology to realize length standards is also one of our most important missions, on which we have long operated the national measurement standards to achieve the SI meter. From a historical perspective, researchers have traditionally attempted to realize length standards, while optical frequency standards only came to be required at the beginning of the $21^{\text {st }}$ century. Taking this history into consideration, we would like to mention length standards to begin with, followed by optical frequency standards. Therefore, it would be appreciated if you could allow us to keep the title unchanged.

\section{Application fields of optical fiber combs Comment (Hidemi Tsuchida)}

I understand from the title that the paper focuses on optical communication as the main application field of optical fiber combs. However, chapter 5 concerns diverse topics such as optical communication, length standards, international comparisons, and high-speed control. It may be reasonable to pick up a topic on length standards, but I recommend enriching the discussions on optical communication. For the purpose of helping the readers' understanding, it will be necessary to include descriptions on the frequency grid standardized in optical communication, the demands for frequency measurement technology, and how fiber combs are utilized in the industry.

Answer (Hajime Inaba, Atsushi Onae, and Feng-Lei Hong)

We believed that in discussing the achievements of the fiber comb in this paper, the various applications would also be an important point. Nevertheless, we have added further descriptions of optical communication at the beginning of chapter 5 to prevent readers from feeling that the topic of the paper is spread out as has been pointed out.

\section{Motivation and predictions of fiber comb development Question and comment (Naoto Kobayashi)}

This paper argues that the use of the fiber combs overcame almost all drawbacks of the solid-state laser comb and that it successfully created an optical frequency comb of extremely high precision. Now, I have two questions. (1) While the paper describes that the motivation for developing fiber combs was a sophisticated fiber laser provided by a joint research company, did you begin by anticipating that the development of the fiber comb could result in a more sophisticated comb? (2) In relation to the description that selffabrication of fiber combs was the major development factor on this occasion, did you anticipate from the beginning that this would cause the development process to proceed well?

Answer (Hajime Inaba, Atsushi Onae, and Feng-Lei Hong)

We conducted joint research with a company as part of the 2002-2004 Project of the Special Coordination Funds for Promoting Science and Technology called "Broadband Optical Synthesizer". The mode-locked fiber laser from that company was reliable and superior in the sense that the incorporated nonlinear crystal was able to generate an optical frequency comb in the 800-nm band. Furthermore, in 2004, we achieved absolute optical frequency measurement in combination with the highly nonlinear fiber, when development of the fiber comb slowed.

However, as the laser system was a product developed by the company, we could not alter the component that generated the comb but instead had to ask the company for alterations, for instance, when the $\mathrm{S} / \mathrm{N}$ ratio of the beat signal was low or when we were dissatisfied with the control or other systems. We also had a number of studies we wanted to engage in using the optical frequency comb, which meant that we needed a large quantity of them. Under these circumstances, we realized the importance of self-fabrication of all systems, including selection of mode-locked fiber lasers, amplifier systems, and highly nonlinear fibers in order to perform the subsequent development and use it as a tool to advance the study.

With experience in fabricating Ti:S combs and vendor fiber combs and in fabricating CW fiber lasers, we were at least certain we could self-fabricate a system for frequency measurement. However, we were aware of a number of challenges to overcome. We were not confident enough to produce the most sophisticated and easy-to-use self-fabricated systems, however.

We still have some opportunities to examine commercially available comb systems, which are often designed to make it harder for us to alter on our own, even if they contain unwanted functions. We would not be willing to use those troublesome systems.

\section{Realization of high performance optical frequency comb} Question and comment (Hidemi Tsuchida)

At the beginning of section 4.1 there is a description on performance comparison (power and pulse width) between the Ti:S laser and fiber laser, but it does not clearly state how the difference in the performances were overcome in order to meet the specification as an optical frequency comb. Please describe the method used (e.g., optical amplification, pulse compression) and to what extent the difference reduced.

\section{Answer (Hajime Inaba)}

In broadening the optical frequency comb, not only was it effective to improve the laser performances like power or pulse width, but it was also necessary to optimize components other than the laser by selecting the highly nonlinear fiber or elaborating the CEO signal detection system. On this occasion, for instance, we successfully detected the CEO signal by detecting the CEO signal of a comb with a wavelength band less than an octave, by means of a $2 \mathrm{f}-3 \mathrm{f}$ interferometer or by finding and applying the optimal highly nonlinear fiber. As added in section 4.5, the differences in the laser performances like power or pulse width in the time domain were no smaller than those of the Ti:S comb, owing to the self-fabrication of the laser. What we intend to develop is a fiber comb that is robust and has low noise, and is sophisticated and practical in the frequency domain.

\section{Realization of high performance optical frequency comb Question and comment (Hidemi Tsuchida)}

Section 4.3 describes the necessity of dispersion control, which seems to be common knowledge for researchers in optical fiber communication or ultrashort optical pulses. The difference between the mean and peak powers is not clear and their relationship with spectral broadening is not clarified.. Please revise the corresponding descriptions intelligibly including the cause of the accompanying power enhancement.

Answer (Hajime Inaba)

We have differentiated the mean power from the peak power as pointed out. We also added a possible cause for the power boost although this is a hypothesis. The phenomenon discussed in section 4.3 regarding distributed control-in that the mean power of radiation increases by properly adjusting the chirp quantity of optical pulses entering the optical amplifier-is our original result supported by academic papers and patents. It would be appreciated if you could kindly examine this. 